Pacific Journal of Mathematics

BOUNDARY VALUE PROBLEMS FOR A CLASS OF
NONLINEAR DIFFERENTIAL EQUATIONS 


\title{
BOUNDARY VALUE PROBLEMS FOR A CLASS OF NONLINEAR DIFFERENTIAL EQUATIONS
}

\author{
GerAld H. RYdeR
}

For certain functions $f$, positive in $(0, \infty)$ and continuous in $[0, \infty)$, the partial differential equation $\Delta x=x-x f\left(x^{2}\right)$ has spherically symmetric solutions $x_{n}(t), n=1,2, \cdots$, which vanish at zero, infinity and $n-1$ distinct values in $(0, \infty)$. This and similar existence theorems for the ordinary differential equation $\ddot{y}-y+y F\left(y^{2}, t\right)=0$ are proved by way of variational problems and the solutions are thus characterized by associated "eigenvalues". The asymptotic behavior of these eigenvalues is studied and some numerical data on the solutions is furnished for special cases of the above equations which are of interest in nuclear physics.

We begin by considering differential equations of the form

$$
\ddot{y}-y+y F\left(y^{2}, t\right)=0,
$$

were $F(\eta, t)$ satisfies the following conditions:

(Ia) $F(\eta, t)$ is continuous in $\eta$ and $t$ for $0<t<\infty$ and $0 \leqq \eta<\infty$;

(Ib) $F(\eta, t)>0$ for $\eta>0, t>0$;

(Ic) there exists a $\delta>0$ such that, for every fixed positive $t$ and $0 \leqq \eta_{1}<\eta_{2}<\infty, \gamma_{2}^{-\delta} F\left(\eta_{2}, t\right)>\eta_{1}^{-\delta} F\left(\eta_{1}, t\right)$.

In the special case in which $F\left(y^{2}, t\right)=f\left(y^{2} / t^{2}\right)$, the substitution

$$
x(t)=t^{-1} y(t)
$$

transforms equation (1.1) into the form

$$
\ddot{x}+\frac{2 \dot{x}}{t}=x-x f\left(x^{2}\right),
$$

which is satisfied by spherically symmetric solutions of the partial differential equation

$$
\Delta x=x-x f\left(x^{2}\right),
$$

where $\Delta$ is the three-dimensional Laplace operator and $t$ denotes distance from the origin.

To simplify our statements concerning solutions of (1.1) and (1.3), we shall employ the following terminology.

Definition I. A solution $y(t)$ of equation (1.1) which is continuous in $[0, \infty)$, positive in $(0, \infty)$, and satisfies $y(0)=0, \lim _{t \rightarrow \infty} y(t)=0$, shall be called a fundamental solution of (1.1) for the interval $[0, \infty)$. 
Definition II. A solution $x(t)$ of equation (1.3) which is continuous in $[0, \infty)$, positive in $(0, \infty)$, and satisfies $\lim x(t)=0$ shall be called a fundamental solution of (1.3) for the interval $[0, \infty)$.

Special cases of equations (1.1) and (1.3) have been studies by a number of authors $[1,3,7,9]$ in connection with problems in nuclear physics, and the existence of fundamental solutions for the interval $[0, \infty)$ was suggested by physical considerations when (1.1) is of the type

$$
\ddot{y}-y+y \frac{|y|^{k-1}}{t^{k-1}}=0
$$

and $k=2,3$. Nehari [4] has shown that such solutions do in fact exist whenever $1<k<5$. In addition, $\lim t^{-1} y(t)$ exists as $t \rightarrow 0$ for $1<k \leqq 4$. This shows that, in view of $(1.2)$, the equation

$$
\ddot{x}+\frac{2 \dot{x}}{t}=x-x|x|^{k-1}
$$

has fundamental solutions whenever $1<k \leqq 4$. Synge [8] also studied equations (1.5) and (1.6) for $k=2$ and set up a numerical procedure for the calculation of $y(t)$ and $x(t)$. Although no proof was given that the procedure converges to a solution, Synge's numerical results were accurate, as we shall see in $\S 8$.

Our purpose is to prove the existence of not only fundamental solutions to equations (1.1) and (1.3), but also solutions $y_{n}(t)$ and $x_{n}(t)$ possessing $(n-1)$ distinct zeros $t_{1}, t_{2}, \cdots, t_{n-1}$ in $(0, \infty)$ and which are such that $y_{n}(t)$ and $x_{n}(t)$ do not vanish in $\left(t_{\nu}, t_{\nu+1}\right), \nu=0,1, \cdots, n-1$ $\left(t_{0}=0, t_{n}=\infty\right)$. Such solutions which change sign as $t \rightarrow \infty$ are again suggested by physical considerations for the case $f\left(x^{2}\right)=x^{2}$ in (1.3) [3].

We shall establish the following result.

THEOREM I. If $F(\eta, t)$ satisfies conditions (Ia)-(Ic) and, in addition,

(Id) $\lim _{t \rightarrow \infty} F\left(c^{2}, t\right)=0$ for all finite $c$,

(Ie) $\int_{0}^{a} t^{(1 / 2)-\varepsilon} F\left(c^{2} t, t\right) d t<+\infty$ for all finite $c, 0<a<\infty$, and some $\varepsilon \geqq 0$,

then equation (1.1) has a discrete infinity of solutions $\left\{y_{n}(t)\right\}, n=$ $1,2, \cdots$, whose derivatives are continuous throughout $[0, \infty)$ and are such that $y_{n}(t)$ has exactly $(n-1)$ zeros in $(0, \infty)$. Moreover, $y_{n}(0)=0$, $\lim t^{-1} y_{n}(t)$ exists as $t \rightarrow 0$ and $y_{n}(t) \rightarrow 0$ as $t \rightarrow \infty$, for each $n$.

Since condition (Ie) is not satisfied for $F\left(y^{2}, t\right)=\left(|y|^{k-1}\right) / t^{k-1}$ when $4 \leqq k<5$, the known existence of fundamental solutions to equation 
(1.5) for $1<k<5$ suggests that, when it is not required that $\lim t^{-1} y(t)$ exist as $t \rightarrow 0$, condition (Ie) may be relaxed to assume the form

(If) $\int_{0}^{a} t^{-\varepsilon} F\left(c^{2} t, t\right) d t<\infty$ for some $\varepsilon>0$ and all finite $c, 0<a<\infty$. Indeed, such is the case at least when $F\left(y^{2}, t\right)=p(t) y^{2 \alpha}$, and we can prove a result similar to Theorem $I$ for the equation

$$
\ddot{y}-y+p(t) y^{2 \alpha+1}=0
$$

when the following conditions (equivalent to (Ia)-(Id) and (If) are satisfied.

$\left(\mathrm{Ia}^{\prime}\right) \quad p(t)$ is continuous in $(0, \infty)$;

$\left(\mathrm{Ib}^{\prime}\right) \quad p(t)>0$ for all $t>0$;

(Ic') $\quad \alpha>0$

$(\mathrm{Id}) \quad \lim _{t \rightarrow \infty} p(t)=0$

( $\left.\mathrm{Ie}^{\prime}\right) \quad \int_{0}^{a} t^{1-\varepsilon+\alpha} p(t) d t<+\infty$ for some $\varepsilon>0$ and $0<a<\infty$.

It is easy to verify that conditions $\left(\mathrm{Ia}^{\prime}\right)-\left(\mathrm{Ie}^{\prime}\right)$ are satisfied for $p(t) y^{2 \alpha}=\left(|y|^{k-1}\right) / t^{k-1}$ and $1<k<5$. (i.e., for $\alpha=(k-1) / 2, p(t)=$ $\left.1 / t^{k-1}\right)$ if we let $\varepsilon=(5-k) / 4$. This is in agreement with the results stated for equation (1.5). Moreover, it was shown in [4] that no fundamental solution to (1.5) can exist for $k=5$.

Finally, in the special case in which (1.1) reduces to (1.3), Theorem I takes the following form.

THEOREM II. If $f(\eta)$ satisfies the conditions:

(IIa) $f(\eta)$ is continuous for $0 \leqq \eta<\infty$,

(IIb) $f(\eta)>0$ for $\eta>0$,

(IIc) $\eta_{2}^{-\delta} f\left(\eta_{2}\right)>\eta_{1}^{-\delta} f\left(\eta_{1}\right)$ for $0 \leqq \eta_{1}<\eta_{2}<\infty$ and some positive $\delta$,

(IId) $\int_{a}^{\infty} \eta^{\varepsilon-(5 / 2)} f(\eta) d \eta<+\infty$ for some $a>0$ and $\varepsilon \geqq 0$.

then equation (1.3) has solutions $x_{n}(t), n=1,2, \cdots$, whose derivatives are continuous in $(0, \infty)$, are such that $\lim x_{n}(t)$ exists as $t \rightarrow 0$, $x_{n}(t) \rightarrow 0$ as $t \rightarrow \infty$, and $x_{n}(t)$ has exactly $n-1$ zeros in $(0, \infty)$.

This result is merely a corollary to Theorem I where the condition corresponding to (Id) is automatically satisfied whenever (IIc) is true. Indeed, by (IIc),

$$
f\left(\frac{c^{2}}{t^{2}}\right)<\frac{t_{0}^{2 \delta}}{t^{2 \delta}} f\left(\frac{c^{2}}{t_{0}^{2}}\right) \text { for finite } t_{0}, t>t_{0}
$$

and thus, for fixed $t_{0}>0$, we have 


$$
f\left(\frac{c^{2}}{t^{2}}\right) \leqq K \frac{1}{t^{2 \delta}} \text { for fixed positive } K, t>t_{0}
$$

In addition to proving the above existence theorems, we shall show that the solutions to (1.1) and (1.7) are characterized by a minimum problem and associated characteristic values $\lambda_{n}$. For $\alpha=1, p(t)=$ $1 / t^{2}$ in (1.7), i.e., when $y(t)$ satisfies

$$
\ddot{y}-y+\frac{y^{3}}{t^{2}}=0,
$$

we shall calculate $\lambda_{1}$ and find bounds for the asymptotic values of the $n^{\text {th }}$ "eigenvalues" $\lambda_{n}$ which are defined by

$$
\lambda_{n}=\frac{1}{2} \int_{0}^{\infty}\left(\dot{y}_{n}^{2}+y_{n}^{2}\right) d t .
$$

By converting the existence proof into a numerical procedure for computing the fundamental solutions of (1.5), we abtain additional numerical information concerning the solutions to (1.9) and the corresponding equation

$$
\ddot{x}+\frac{2 \dot{x}}{t}=x-x^{3} .
$$

Both (1.9) and (1.10) were studied by Mitskevich [3].

2. A minimum problem. As a first step in the proof of Theorem I, we show that equation (1.1) has a fundamental solution for which $\lim _{t \rightarrow 0} t^{-1} y(t)$ exists when $F(\eta, t)$ satisfies the stated conditions. To do this, we shall set up a variational problem as in [5] and show that this problem has a solution which must satisfy (1.1) and the boundary conditions for a fundamental solution. We consider the problem

$$
J(y)=\int_{0}^{\infty}\left[\dot{y}^{2}+y^{2}-G\left(y^{2}, \tau\right)\right] d \tau=\min .
$$

where $y(t)$ is subject to the admissibility conditions $y(0)=0, y(t) \not \equiv 0$ in $(0, \infty), y(t) \geqq 0$ in $[0, \infty), y(t) \in D^{\prime}[0, \infty)$, and the normalization condition

$$
\int_{0}^{\infty}\left(\dot{y}^{2}+y^{2}\right) d \tau=\int_{0}^{\infty} y^{2} F\left(y^{2}, \tau\right) d \tau .
$$

The function $G\left(y^{2}, \tau\right)$ appearing in (2.2) is defined by

$$
G\left(y^{2}, \tau\right)=\int_{0}^{y^{2}} F(\eta, \tau) d \eta, \text { for each } \tau \text { in }(0, \infty) .
$$


Because of conditions (Ib) and (Ic), it can be shown that any function $y(t)$ satisfying the admissibility conditions, and for which $\int_{0}^{\infty}\left(\dot{y}^{2}+y^{2}\right) d \tau$ exists, can be multiplied by a positive constant $\alpha$ such that $\alpha y(t)$ satisfies (2.2). We first show that the existence of $\int_{0}^{\infty}\left(\dot{y}^{2}+y^{2}\right) d \tau$ implies that of $\int_{0}^{\infty} y^{2} F\left(y^{2}, \tau\right) d \tau$.

Setting $\int_{0}^{\infty}\left(\dot{y}^{2}+y^{2}\right) d \tau=\sigma^{2}$ and noting that $y(0)=0$, we have

$$
\begin{gathered}
y^{2}(t)=\left[\int_{0}^{t} \dot{y} d \tau\right]^{2} \leqq t \int_{0}^{t} \dot{y}^{2} d \tau \leqq t \sigma^{2} \\
y^{2}(t)=2 \int_{0}^{t} y \dot{y} d \tau \leqq \int_{0}^{t}\left(\dot{y}^{2}+y^{2}\right) d \tau \leqq \sigma^{2} .
\end{gathered}
$$

Hence, taking some $t_{0}$ in $(0, \infty), T>t_{0}, \varepsilon \geqq 0$, and using (2.4) in $\left[0, t_{0}\right]$ and $(2.5)$ in $\left[t_{0}, T\right]$, we have

$$
\int_{0}^{T} y^{2} F\left(y^{2}, \tau\right) d \tau \leqq \sigma^{2} t_{0}^{\varepsilon} \int_{0}^{t_{0}} \tau^{1-\varepsilon} F\left(\sigma^{2} \tau, \tau\right) d \tau+\max _{t_{0} \leqq t<\infty}\left[F\left(\sigma^{2}, t\right)\right] \int_{t_{0}}^{T} y^{2} d \tau .
$$

This shows that

$$
\int_{0}^{T} y^{2} F\left(y^{2}, \tau\right) d \tau \leqq M_{1}\left(\sigma^{2}\right) \sigma^{2}+M_{2}\left(\sigma^{2}\right) \sigma^{2},
$$

where $M_{1}\left(\sigma^{2}\right)=t_{0}^{\varepsilon} \int_{0}^{t_{0}} \tau^{1-\varepsilon} F\left(\sigma^{2} \tau, \tau\right) d \tau$ and $M_{2}\left(\sigma^{2}\right)=\max _{t_{0} \leqq t<\infty}\left[F\left(\sigma^{2}, t\right)\right]$ are both finite for all finite $\sigma^{2}$ when $F$ satisfies (Id) and either (If) or (Ie).

To complete the proof that $y(t)$ may be normalized as in equation (2.2), we define

$$
B(\alpha)=\frac{\int_{0}^{\infty}\left(\dot{y}^{2}+y^{2}\right) d \tau}{\int_{0}^{\infty} y^{2} F\left(\alpha^{2} y^{2}, \tau\right) d \tau}
$$

If $\alpha>1,\left[F\left(\alpha^{2} y^{2}, t\right)\right] /\left(\alpha^{2} y^{2}\right)^{\delta}>\left[F\left(y^{2}, t\right)\right] / y^{2 \delta}$ by (Ic), and thus

$$
B(\alpha)<\frac{\int_{0}^{\infty}\left(\dot{y}^{2}+y^{2}\right) d \tau}{\alpha^{2} \int_{0}^{\infty} y^{2} F\left(y^{2}, \tau\right) d \tau}, \quad \alpha>1 .
$$

If $\alpha<1,\left[F\left(y^{2}, t\right)\right] / y^{2 \delta}>\left[F\left(\alpha^{2} y^{2}, t\right)\right] /\left(\alpha^{2} y^{2}\right)^{\delta}$, and

$$
B(\alpha) \geqq \frac{\int_{0}^{\infty}\left(\dot{y}^{2}+y^{2}\right) d \tau}{\alpha^{2} \int_{0}^{\infty} y^{2} F\left(y^{2}, \tau\right) d \tau}, \quad \alpha \leqq 1 .
$$

Because of conditions (Ia), (Ic) and the fact $B(\alpha) \rightarrow 0$ as $\alpha \rightarrow \infty$ by 
(2.8) and $B(\alpha) \rightarrow \infty$ as $\alpha \rightarrow 0$ by (2.9), the continuous function (of $\alpha$ ) $B(\alpha)$ assumes all values in $(0, \infty)$ as $\alpha$ varies in this same range. In particular $B(\alpha)=1$ for some $\alpha$ in $(0, \infty)$ and (2.7) shows that $v(t)=$ $\alpha y(t)$ consequently satisfies

$$
\int_{0}^{\infty}\left(\dot{v}^{2}+v^{2}\right) d \tau=\int_{0}^{\infty} v^{2} F\left(v^{2}, \tau\right) d \tau .
$$

Finally, we see that

$$
\int_{0}^{T} G\left(y^{2}, \tau\right) d \tau \leqq \int_{0}^{T} y^{2} F\left(y^{2}, \tau\right) d \tau \quad \text { for } T \in(0, \infty)
$$

by definition of $G\left(y^{2}, \tau\right)$ and thus, in view of (2.6) and (2.10), the existence of the integral in (2.1) is also assured whenever $\int_{0}^{\infty}\left(\dot{y}^{2}+y^{2}\right) d \tau$ exists.

3. Associated comparison functions. To show that our variational problem has a nontrivial solution we employ some of the techniques of Nehari [5], where such functions $F(\eta, t)$ satisfying (Ia) - (Ic) were considered, and [4], where the differential equation (1.5) with singularities at zero and infinity was studied.

By (Ic) and (2.3) we have

$$
G(\eta, t)=\int_{0}^{\eta} x^{\delta}\left[x^{-\delta} F(x, t)\right] d x \leqq \eta^{-\delta} F(\eta, t) \int_{0}^{\eta} x^{\delta} d x=\frac{\eta}{1+\delta} F(\eta, t) .
$$

Hence,

$$
\eta F(\eta, t)-G(\eta, t) \geqq \delta(1+\delta)^{-1} \eta F(\eta, t),
$$

and, if $y(t)$ is an admissible function satisfying (2.2), this inequality shows that

$$
J(y) \geqq \delta(1+\delta)^{-1} \int_{0}^{\infty}\left(\dot{y}^{2}+y^{2}\right) d \tau .
$$

Furthermore, if for all admissible $y(t)$,

$$
\lambda=\text { g.l.b. } J(y),
$$

there will exist a sequence of functions $y_{1}(t), y_{2}(t), \cdots$, which satisfy the conditions of the minimum problem (2.1), (2.2), and for which

$$
\lim _{n \rightarrow \infty} J\left(y_{n}\right)=\lambda \geqq 0 \text {. }
$$

The fact that $\lambda \geqq 0$ follows from (3.1).

It also follows from (3.1) that such a sequence $\left\{y_{n}(t)\right\}$ is uniformly bounded and equicontinuous in every finite interval $[0, T]$. Indeed, 


$$
\int_{0}^{\infty}\left(\dot{y}_{n}^{2}+y_{n}^{2}\right) d \tau \leqq \rho^{2}<\infty
$$

for some positive constant $\rho$ and, by (2.5), this shows that

$$
y_{n}^{2}(t) \leqq \rho^{2} \quad \text { for all } t \text { in }[0, \infty), n=1,2, \cdots,
$$

Moreover, using (3.4), we have

$$
\begin{aligned}
\left|y_{n}\left(t_{2}\right)-y_{n}\left(t_{1}\right)\right|^{2} & =\left[\int_{t_{1}}^{t_{2}} \dot{y}_{n} d t\right]^{2} \leqq\left(t_{2}-t_{1}\right) \int_{t_{1}}^{t_{2}} \dot{y}_{n}^{2} d t \\
& \leqq \rho^{2}\left(t_{2}-t_{1}\right)
\end{aligned}
$$

for all $0 \leqq t_{1}<t_{2}<\infty$.

By Ascoli's Lemma, there exists a subsequence of $\left\{y_{n}(t)\right\}$ which converges, uniformly on every finite interval $[0, T]$, to a continuous function $y(t)$. We have thus proved the following result:

Lemma (3.1). There exists a sequence $\left\{y_{n}(t)\right\}$ of functions, admissible for the variational problem (2.1), (2.2), which converges uniformly in every finite interval [0,T] to a continuous function $y(t)$. Moreover, $\lim _{n \rightarrow \infty} J\left(y_{n}\right)=\lambda=$ g.l.b. $J(y) \geqq 0$.

What we now wish to show is that, for each $y_{n}(t)$ defined above and $\alpha_{n}$ constant, the linear differential equation

$$
\ddot{u}_{n}-u_{n}+\alpha_{n} y_{n} F\left(y_{n}^{2}, t\right)=0
$$

has a solution satisfying $u_{n}(0)=0, \lim _{t \rightarrow \infty} u_{n}(t)=0$. Moreover, this solution is also an admissible function (for suitable $\alpha_{n}$ ) and

$$
J\left(u_{n}\right) \leqq J\left(y_{n}\right) .
$$

To do this, we consider the integral equation corresponding to (3.6):

$$
u_{n}(t)=\alpha_{n} \int_{0}^{\infty} g(t, \tau) y_{n}(\tau) F\left(y_{n}^{2}, \tau\right) d \tau
$$

where $g(t, \tau)$ is the Green's function of the differential operator $L(u)=$ $\ddot{u}-u$ for the boundary conditions $u(0)=0, \lim _{t \rightarrow \infty} u(t)=0$, and is defined by

$$
g(t, \tau)= \begin{cases}\bar{e}^{t} \sinh \tau, & 0 \leqq \tau<t \\ \bar{e}^{\tau} \sinh t, & t \leqq \tau\end{cases}
$$

Under the conditions imposed on $F(\eta, t)$ and the admissibility conditions imposed on $y_{n}(t)$, we shall prove that $u_{n}(t)$ defined in (3.8) is indeed the desired solution of (3.6). 
Using (3.9), we see that (temporarily setting $\alpha_{n}=1$ )

$$
\begin{aligned}
u_{n}(t)= & \bar{e}^{t} \int_{0}^{t} \sinh \tau y_{n} F\left(y_{n}^{2}, \tau\right) d \tau \\
& +\sinh t \int_{t}^{\infty} \bar{e}^{\tau} y_{n} F\left(y_{n}^{2}, \tau\right) d \tau .
\end{aligned}
$$

Employing the definitions

$$
\begin{aligned}
& Q(t)=\int_{0}^{t} \sinh \tau y_{n} F\left(y_{n}^{2}, \tau\right) d \tau \\
& R(t)=\int_{t}^{\infty} \bar{e}^{\tau} y_{n} F\left(y_{n}^{2}, \tau\right) d \tau,
\end{aligned}
$$

equation (3.10) takes the form (for $\alpha_{n}=1$ )

$$
u_{n}(t)=\bar{e}^{t} Q(t)+R(t) \sinh t .
$$

To study the behaviour of $Q(t)$ near zero, we use (2.4), (3.4) and the monotonicity of $t^{\varepsilon-(1 / 2)} \sinh t$ in $(0, t)$ for $\varepsilon \geqq 0$. Equation (3.11) yields

$$
Q(t) \leqq \rho t^{\varepsilon-(1 / 2)} \sinh t \int_{0}^{t} \tau^{1-\varepsilon} F\left(\rho^{2} \tau, \tau\right) d \tau, \quad \varepsilon \geqq 0 .
$$

On the other hand, we see from (3.12) that for $0<t<1$

$$
\begin{aligned}
R(t) & =R(1)+\int_{t}^{1} e^{-\tau} y_{n} F\left(y_{n}^{2}, \tau\right) d \tau \\
& <R(1)+\rho \int_{t}^{1} \tau^{1 / 2} F\left(\rho^{2} \tau, \tau\right) d \tau,
\end{aligned}
$$

where $R(1) \leqq \rho e^{-1}\left[\max _{1 \leq t<\infty} F\left(\rho^{2}, t\right)\right]$.

Since $\tau^{\varepsilon-(1 / 2)}<t^{\varepsilon-(1 / 2)}$ for $\tau>t, 0 \leqq \varepsilon \leqq 1 / 2$, and since $\tau^{\varepsilon-(1 / 2)} \leqq 1$ for $0<\tau<1, \varepsilon \geqq 1 / 2$, the last inequality becomes

$$
R(t) \leqq R(1)+ \begin{cases}\rho \int_{t}^{1} \tau^{1-\varepsilon} F\left(\rho^{2} \tau, \tau\right) d \tau, & \varepsilon \geqq 1 / 2 \\ \rho t^{\varepsilon-(1 / 2)} \int_{t}^{1} \tau^{1-\varepsilon} F\left(\rho^{2} \tau, \tau\right) d \tau, & 0 \leqq \varepsilon \leqq 1 / 2 .\end{cases}
$$

If we combine (3.13), (3.14) and (3.15) we see that $u_{n}(t) \rightarrow 0$ as $t \rightarrow 0$ provided $F(\eta, t)$ satisfies (If). If (Ie) is fulfilled and we use this condition in the equivalent form

$$
\int_{0}^{a} \tau^{1-\varepsilon} F\left(c^{2} \tau, \tau\right) d \tau<\infty, \quad \varepsilon \geqq 1 / 2
$$

then $t^{-1} u_{n}(t)$ approaches a finite limit as $t \rightarrow 0$.

To study the behavior of $Q(t)$ and $R(t)$ for large $t$ we use (2.5) 
and (3.4) in (3.12) to find that, for $t>0$

$$
R(t) \leqq \rho e^{-t}\left[\max _{t \leqq \tau<\infty} F\left(\rho^{2}, \tau\right)\right] .
$$

Also, if $0<t_{0}<t$,

$$
Q(t)=Q\left(t_{0}\right)+\int_{t_{0}}^{t} \sinh \tau y_{n} F\left(y_{n}^{2}, \tau\right) d \tau
$$

where $Q\left(t_{0}\right)$ is finite by $(3.14)$.

In view of (2.5) and (3.4), we then have

$$
Q(t) \leqq Q\left(t_{0}\right)+\frac{\rho}{2} \int_{t_{0}}^{t} e^{\tau} F\left(\rho^{2}, \tau\right) d \tau, \quad 0<t_{0}<t
$$

Thus, if (Id) is satisfied, $\int_{t_{0}}^{t} e^{\tau} F\left(\rho^{2}, \tau\right) d \tau=o\left(e^{t}\right)$ as $t \rightarrow \infty$ and (3.13), (3.16) and (3.17) show that $u_{n}(t) \rightarrow 0$ as $t \rightarrow \infty$.

We shall now examine the behavior of $\dot{u}_{n}(t)$. Using (3.10) to compute $h^{-1}\left[u_{n}(t+h)-u_{n}(t)\right]$ and letting $h \rightarrow 0$, we see that $\dot{u}_{n}(t)$ exists and is given by

$$
\dot{u}_{n}(t)=-e^{-t} Q(t)+\cosh t R(t) .
$$

Equations (3.16) and (3.17) then show that $\dot{u}_{n}(t) \rightarrow 0$ as $t \rightarrow \infty$ whenever $F(\eta, t)$ satisfies (Id) and either (Ie) or (If). Similarly, (3.14) and (3.15) show that $\left|\dot{u}_{n}(t)\right|$ is bounded near $t=0$ if $F$ satisfies (Id) and (Ie). If only (If) is satisfied, then it is seen $\dot{u}_{n}(t)=0\left(t^{\varepsilon-(1 / 2)}\right)$ as $t \rightarrow 0$.

If we now compute $h^{-1}\left[\dot{u}_{n}(t+h)-\dot{u}_{n}(t)\right]$ from (3.18), we find that $\ddot{u}_{n}(t)$ exists and that $u_{n}(t)$ is a solution of (3.6) for $\alpha_{n}=1$. Moreover, $u_{n}(t)$ is nonnegative in $(0, \infty)$ since $y_{n}(t)$ was assumed to be. Finally, we can show that

$$
\lim _{t \rightarrow 0} \dot{u}_{n}(t) u_{n}(t)=0,
$$

if we combine (3.18) with the above comments concerning $\dot{u}_{n}(t)$. We may summarize our results as follows:

Lemma 3.2. If $y_{n}(t)$ is defined as in Lemma 2.1 and $\alpha_{n}$ is a constant, then equation (3.6) has a solution $u_{n}(t)$ satisfying $u_{n}(0)=0$, $\lim _{t \rightarrow \infty} u_{n}(t)=0$ whenever $F(\eta, t)$ satisfies (Ia)-(Id) and (If) or (Ie). Moreover $u_{n}(t)$ is such that $\lim _{t \rightarrow \infty} \dot{u}_{n}(t)=0, \lim _{t \rightarrow 0} u_{n}(t) \dot{u}_{n}(t)=0$ and, if condition (Ie) is fulfilled, $\lim _{t \rightarrow 0} t^{-1} u_{n}(t)$ exists.

4. Convergence of the comparison functions to a fundamental solution. We now proceed to prove the existence of a fundamental solution to (1.1). To do this we first show that, for suitable $\alpha_{n}, u_{n}(t)$ 
is an admissible function for our variational problem and satisfies (3.7).

Multiplying (3.6) by $u_{n}(t)$, integrating from 0 to $T$, and using (3.19), we obtain

$$
\sigma_{1}^{2}(T)=\int_{0}^{T}\left(\dot{u}_{n}^{2}+u_{n}^{2}\right) d \tau=\alpha_{n} \int_{0}^{T} u_{n} y_{n} F\left(y_{n}^{2}, \tau\right) d \tau+u_{n}(T) \dot{u}_{n}(T) .
$$

Using (2.5) to estimate $u_{n}(t)$ and (3.5) to estimate $y_{n}(t)$ in the interval $[0,1]$, and employing the Schwarz inequality in $[1, T]$, we find that

$$
\begin{aligned}
& \int_{0}^{T} u_{n} y_{n} F\left(y_{n}^{2}, \tau\right) d \tau \leqq \sigma_{1}(T) \rho \int_{0}^{1} \tau^{1-\varepsilon} F\left(\rho^{2} \tau, \tau\right) d \tau \\
& +\left[\int_{1}^{T} u_{n}^{2} F\left(y_{n}^{2}, \tau\right) d \tau\right]^{1 / 2}\left[\int_{1}^{T} y_{n}^{2} F\left(y_{n}^{2}, \tau\right) d \tau\right]^{1 / 2} .
\end{aligned}
$$

Hence, in view of (2.2) (applied to $\left.y_{n}(t)\right)$ and (3.4),

$$
\int_{0}^{T} u_{n} y_{n} F\left(y_{n}^{2}, \tau\right) d \tau \leqq \rho c_{1} \sigma_{1}(T)+\rho \sigma_{1}(T)\left[\max _{1 \leqq t<\infty}\left\{F\left(\rho^{2}, t\right)\right\}\right]^{1 / 2},
$$

where $c_{1}=\int_{0}^{1} \tau^{1-\varepsilon} F\left(\rho^{2} \tau, \tau\right) d \tau$ and $\max _{1 \leqq t<\infty}\left\{F\left(\rho^{2}, t\right)\right\}$ exist by properties (Id), and (If) or (Ie).

Combining (4.1) and (4.2), it follows that

$$
\sigma_{1}^{2}(T) \leqq \alpha_{n} c_{2} \sigma_{1}(T)+u_{n}(T) \dot{u}_{n}(T),
$$

where $c_{2}$ is a constant independent of $n$. Completing the square in the last inequality, we have

$$
\left[\sigma_{1}(T)-\frac{\alpha_{n} c_{2}}{2}\right]^{2} \leqq \frac{\alpha_{n}^{2} c_{2}^{2}}{4}+u_{n}(T) \dot{u}_{n}(T)
$$

However, since $u_{n}(T)$ and $\dot{u}_{n}(T)$ tend to zero as $T \rightarrow \infty$ (Lemma 3.2), this establishes the existence of the integral $\int_{0}^{\infty}\left(\dot{u}_{n}^{2}+u_{n}^{2}\right) d \tau$ and, because of (2.7), also the existence of $\int_{0}^{\infty} u_{n}^{2} F\left(u_{n}^{2}, \tau\right) d \tau$. Therefore, as shown in $\S 2$, we may choose the constant $\alpha_{n}$ in such a way that

$$
\int_{0}^{\infty}\left(\dot{u}_{n}^{2}+u_{n}^{2}\right) d \tau=\int_{0}^{\infty} u_{n}^{2} F\left(u_{n}^{2}, \tau\right) d \tau \text {. }
$$

and $u_{n}(t)$ becomes an admissible function for the problem (2.1), (2.2). If we use the convexity of $G(\eta, t)$, the Schwarz and other elementary inequalities, it is easy to establish inequality (3.7), i.e.,

$$
J\left(u_{n}\right) \leqq J\left(y_{n}\right) .
$$

Moreover, in view of the way in which these inequalities are used, equality is possible only if $u_{n}$ and $y_{n}$ coincide. If we note that the existence of all integrals involved is insured by the facts that $\dot{u}_{n}(t)=0$ 
$\left(t^{\varepsilon-(1 / 2)}\right)$ and $y_{n}(t)=0\left(t^{1 / 2}\right)$ near zero, the proof proceeds like a comparable one in [5] and will be omitted. The proof also establishes the useful inequality

$$
\alpha_{n}^{2} \int_{0}^{\infty} y_{n}^{2} F\left(y_{n}^{2}, t\right) d t \leqq \int_{0}^{\infty} u_{n}^{2} F\left(u_{n}^{2}, t\right) d t .
$$

Because of the definition of the number $\lambda$ in (3.3), we must have $\lim \inf J\left(u_{n}\right) \geqq \lambda$ since $u_{n}(t)$ is an admissible function. Formulas (4.4) and (3.3) thus lead to the relations

$$
\lim _{n \rightarrow \infty} J\left(u_{n}\right)=\lim _{n \rightarrow \infty} J\left(y_{n}\right)=\lambda .
$$

Moreover, using the same inequalities which lead to the uniform boundedness and equicontinuity of the sequence $\left\{y_{n}(t)\right\}$ in $\S 2$, we find that $\left\{u_{n}(t)\right\}$ converges uniformly in every finite interval $[0, T]$ to a continuous function $u_{0}(t)$, i.e.,

$$
\lim _{n \rightarrow \infty} u_{n}(t)=u_{0}(t) .
$$

This proves:

LEMma 4.1. Under the conditions (Ia)-(Id), and (Ie) or (If), the sequence $u_{n}(t)$ defined in (3.6) converges uniformly in every finite interval $[0, T]$ to a continuous function $u_{0}(t)$. Moreover, $\lim _{n \rightarrow \infty} J\left(u_{n}\right)=\lambda$.

Now, $u_{0}(t)$ will be a solution to our variational problem if we can show that

$$
\lim _{n \rightarrow \infty} J\left(u_{n}\right)=J\left(\lim u_{n}\right)=J\left(u_{0}\right) .
$$

We proceed to establish this result by first proving the uniform convergence of $\dot{u}_{n}(t)$. It is from this point on that we need (Ie) rather than (If) for the existence proof. When $F(\eta, t)$ satisfies (Ie), then, as shown in $\S 3$, each of the $\dot{u}_{n}(t)$ approaches a finite limit as $t \rightarrow 0$. In this case, each $\dot{u}_{n}(t)$ is continuous in [0, ). Furthermore,

$$
\dot{u}_{n}(t)-\dot{u}_{m}(t)=e^{-t}\left[Q_{n}(t)-Q_{m}(t)\right]+\cosh t\left[R_{n}(t)-R_{m}(t)\right]
$$

where $Q_{i}(t), R_{i}(t), i=1,2, \cdots$, were defined in (3.11) and (3.12).

When conditions (Ia)-(Ie) are satisfied, we have shown that each of the $Q_{i}(t), R_{i}(t)$ existed for all $t>0$ as long as $y_{i}(t)$ was a member of $\left\{y_{n}(t)\right\}$. Since the sequence $\left\{y_{n}(t)\right\}$ was shown to converge uniformly in every finite interval $[0, T]$, it is easy to see that the same is true of the sequences $\left\{Q_{n}(t)\right\},\left\{R_{n}(t)\right\}$. Equation (4.9) then shows $\left\{\dot{u}_{n}(t)\right\}$ converges uniformly.

In view of (4.7), therefore, we have 


$$
\lim _{n \rightarrow \infty} \dot{u}_{n}(t)=\dot{u}_{0}(t)
$$

where the convergence is uniform in every finite interval $[0, T]$.

We also need to show that $\alpha_{n}$, which is determined by (4.3), is bounded for all $n$. By (4.5)

$$
\alpha_{n}^{2} \leqq \frac{\int_{0}^{\infty} u_{n}^{2} F\left(u_{n}^{2}, \tau\right) d \tau}{\int_{0}^{\infty} y_{n}^{2} F\left(y_{n}^{2}, \tau\right) d \tau} .
$$

To see that this denominator in (4.11) has a lower bound, we set $\sigma_{n}^{2}=\int_{0}^{\infty}\left(\dot{y}_{n}^{2}+y_{n}^{2}\right) d \tau$ and employ (2.2), (2.4) and (2.5). Thus

$$
\begin{aligned}
\sigma_{n}^{2} & =\int_{0}^{\infty}\left(\dot{y}_{n}^{2}+y_{n}^{2}\right) d \tau=\int_{0}^{\infty} y_{n}^{2} F\left(y_{n}^{2}, \tau\right) d \tau \\
& \leqq \sigma_{n}^{2} \int_{0}^{1} \tau^{1-\varepsilon} F\left(\sigma_{n}^{2} \tau, \tau\right) d \tau+\sigma_{n}^{2} \max _{1 \leqq t<\infty}\left[F\left(\sigma_{n}^{2}, t\right)\right]
\end{aligned}
$$

for $\varepsilon \geqq 0$. Since $\sigma_{n}^{2}>0$ by our requirement that $y_{n}(t) \not \equiv 0$, we can divide both sides of the inequality by $\sigma_{n}^{2}$ and obtain

$$
1 \leqq \int_{0}^{1} \tau^{1-\varepsilon} F\left(\sigma_{n}^{2} \tau, \tau\right) d \tau+\max _{1 \leqq t<\infty}\left[F\left(\sigma_{n}^{2}, t\right)\right]
$$

If it were true that $\sigma_{n}^{2}<1$ for some $n$, condition (Ic) would show that

$$
\begin{aligned}
F\left(\sigma_{n}^{2} \tau, \tau\right) & <\sigma_{n}^{2 \delta} F(\tau, \tau), \\
F\left(\sigma_{n}^{2}, \tau\right) & <\sigma_{n}^{2 \delta} F(1, \tau) .
\end{aligned}
$$

Substituting in (4.13) would then yield

$$
1 \leqq \sigma_{n}^{2 \delta} \int_{0}^{1} \tau^{1-\varepsilon} F(\tau, \tau) d \tau+\sigma_{n}^{2 \delta} \max _{1 \leqq t<\infty}[F(1, t)]
$$

for $\varepsilon \geqq 0$. This inequality shows that $\sigma_{n}^{2}$ cannot approach zero as $n \rightarrow \infty$, i.e.,

$$
\sigma_{n}^{2}=\int_{0}^{\infty} y_{n}^{2} F\left(y_{n}^{2}, \tau\right) d \tau \geqq M>0, \quad \text { for all } n,
$$

when conditions (Ia)-(Id), and (If) or (Ie) are satisfied.

In order to examine the numerator in (4.11), we apply (3.1) to $u_{n}(t)$ and use the normalization (4.3) to obtain

$$
\int_{0}^{\infty} u_{n}^{2} F\left(u_{n}^{2}, \tau\right) d \tau \leqq \frac{1+\delta}{\delta} J\left(u_{n}\right) .
$$


In view of (4.4) and the fact $J\left(y_{n}\right) \leqq \int_{0}^{\infty}\left(\dot{y}_{n}^{2}+y_{n}^{2}\right) d \tau \leqq \rho^{2}$, the last inequality yields

$$
\int_{0}^{\infty} u_{n}^{2} F\left(u_{n}^{2}, \tau\right) d \tau \leqq \frac{1+\delta}{\delta} \rho^{2} .
$$

Combining this result with (4.11) and (4.14), we have

$$
\alpha_{n}^{2} \leqq \frac{1+\delta}{\delta} \frac{\rho^{2}}{M}, \quad \text { for all } n .
$$

Thus $\alpha_{n}$ is bounded above.

To return now to the proof of (4.8), we write

$$
\begin{aligned}
& \left|\int_{0}^{T}\left(\dot{u}_{0}^{2}+u_{0}^{2}\right) d \tau-\int_{0}^{\infty}\left(\dot{u}_{n}^{2}+u_{n}^{2}\right) d \tau\right| \\
& \quad \leqq\left|\int_{0}^{T}\left(\dot{u}_{0}^{2}+u_{0}^{2}\right) d \tau-\int_{0}^{T}\left(\dot{u}_{n}^{2}+\dot{u}_{n}^{2}\right) d \tau\right|+\left|\int_{T}^{\infty}\left(\dot{u}_{n}^{2}+u_{n}^{2}\right) d \tau\right| .
\end{aligned}
$$

In view of (3.6) and Lemma (3.2), however,

$$
\int_{T}^{\infty}\left(\dot{u}_{n}^{2}+u_{n}^{2}\right) d \tau=\alpha_{n} \int_{T}^{\infty} u_{n} y_{n} F\left(y_{n}^{2}, \tau\right) d \tau+u_{n}(T) \dot{u}_{n}(T) .
$$

Moreover,

$$
\begin{aligned}
\int_{T}^{\infty} u_{n} y_{n} F\left(y_{n}^{2}, \tau\right) d \tau & \leqq \max _{T \leqq t<\infty}\left[F\left(\rho^{2}, t\right)\right] \int_{T}^{\infty} u_{n} y_{n} d \tau \\
& \left.\left.\leqq \max _{T \leqq t<\infty}\right] F\left(\rho^{2}, t\right)\right] \rho\left[\int_{T}^{\infty} u_{n}^{2} d \tau\right]^{1 / 2} \\
& \leqq \max _{T \leqq t<\infty}\left[F\left(\rho^{2}, t\right)\right] \rho^{2}\left[\frac{1+\delta}{\delta}\right]^{1 / 2},
\end{aligned}
$$

where the final result follows from the fact that

$$
\int_{0}^{\infty} u_{n}^{2} d \tau \leqq \frac{1+\delta}{\delta} J\left(u_{n}\right) \leqq \frac{1+\delta}{\delta} J\left(y_{n}\right) \leqq \frac{1+\delta}{\delta} \rho^{2} .
$$

If we now combine the above inequality with its predecessor, and substitute into (4.17), we find

$$
\begin{gathered}
\left|\int_{0}^{T}\left(\dot{u}_{0}^{2}+u_{0}^{2}\right) d \tau-\int_{0}^{\infty}\left(\dot{u}_{n}^{2}+u_{n}^{2}\right) d \tau\right| \leqq\left|\int_{0}^{T}\left(\dot{u}_{0}^{2}+u_{0}^{2}\right) d \tau-\int_{0}^{T}\left(\dot{u}_{n}^{2}+u_{n}^{2}\right) d \tau\right| \\
\quad+\alpha_{n} \rho^{2}\left[\frac{1+\delta}{\delta}\right]^{1 / 2} \max _{T \leqq t<\infty}\left[F\left(\rho^{2}, t\right)\right]+\left|u_{n}(T) \dot{u}_{n}(T)\right| .
\end{gathered}
$$

But since

$$
\alpha_{n}\left[\frac{1+\delta}{\delta}\right]^{1 / 2} \rho^{2} \leqq \frac{1+\delta}{\delta} \frac{\rho^{3}}{M} \leqq K
$$


by (4.16), and $\int_{0}^{T}\left(\dot{u}_{n}^{2}+\dot{u}_{n}\right) d \tau$ converges to $\int_{0}^{T}\left(\dot{u}_{0}^{2}+u_{0}^{2}\right) d \tau$ (Lemma 4.1 and the uniform convergence in (4.10)), our last inequality shows that

$$
\begin{aligned}
& \left|\int_{0}^{T}\left(\dot{u}_{0}^{2}+u_{0}^{2}\right) d \tau-\lim _{n \rightarrow \infty} \int_{0}^{\infty}\left(\dot{u}_{n}^{2}+u_{n}^{2}\right) d \tau\right| \\
& \quad \leqq K \max _{T \leqq t<\infty}\left[F\left(\rho^{2}, t\right)\right]+\left|\dot{u}_{0}(T) u_{0}(T)\right| .
\end{aligned}
$$

If we now use the results that $\dot{u}_{0}(T) u_{0}(T) \rightarrow 0$ as $T \rightarrow \infty$ (equation (4.10), Lemma 3.2, Lemma 4.1) and $\max _{T \leqq t<\infty}\left[F\left(\rho^{2}, t\right)\right] \rightarrow 0$ as $T \rightarrow \infty$ (property $(\mathrm{Id})$ ), we finally obtain

$$
\lim _{n \rightarrow \infty} \int_{0}^{\infty}\left(\dot{u}_{n}^{2}+u_{n}^{2}\right) d \tau=\int_{0}^{\infty}\left(\dot{u}_{0}^{2}+u_{0}^{2}\right) d \tau \text {. }
$$

Employing similar techniques, we can also show that

$$
\lim _{n \rightarrow \infty} \int_{0}^{\infty} G\left(u_{n}^{2}, \tau\right) d \tau=\int_{0}^{\infty} G\left(u_{0}^{2}, \tau\right) d \tau .
$$

Hence, equations (4.18), (4.19) and (2.1) yield the result

$$
\lim _{n \rightarrow \infty} J\left(u_{n}\right)=J\left(u_{0}\right),
$$

which proves (4.8) and verifies that $u_{0}(t)$ is indeed the solution of our variational problem. Moreover, since (4.14) also holds for $u_{n}(t)$, equation (4.15) shows that

$$
J\left(u_{n}\right)>\frac{\delta}{1+\delta} M>0 .
$$

Because of $(4.6)$, we have thus proved

$$
\lim _{n \rightarrow \infty} J\left(u_{n}\right)=\lim _{n \rightarrow \infty} J\left(y_{n}\right)=J\left(u_{0}\right)=\lambda>0,
$$

and $u_{0}(t)$ cannot be identically zero in $[0, \infty)$

We now proceed to show that $u_{0}(t)$ satisfies (1.1) and is consequently a fundamental solution for the interval $[0, \infty)$.

As previously remarked, the sign of equality is possible in (4.4) only if $y_{n}(t)$ and $u_{n}(t)$ coincide in $[0, \infty)$. Equation (3.6) shows that, in this case, $y_{n}(t)$ must be a solution of

$$
\ddot{u}-u+\alpha_{u} F\left(u^{2}, t\right)=0 \quad \text { for some } \alpha>0,
$$

where $u(0)=\lim _{t \rightarrow \infty} u(t)=0$. Hence, if we let $y_{n}(t)=u_{0}(t)$, we find

$$
\ddot{u}_{0}-u_{0}+\alpha_{0} u_{0} F\left(u_{0}^{2}, t\right)=0
$$

because of the minimum property of $u_{0}(t)$, i.e., in this case, $J\left(u_{n}\right)=$ 
$J\left(y_{n}\right)$ and the previous comments apply. Furthermore, since $u_{0}(t) \dot{u}_{0}(t) \rightarrow 0$ as $t \rightarrow 0$ and $u_{0}(t) \dot{u}_{0}(t) \rightarrow 0$ as $t \rightarrow \infty$ (equation 4.10 and Lemmas 3.2 and 4.1 , it is also true that

$$
\int_{0}^{\infty}\left(\dot{u}_{0}^{2}+u_{0}^{2}\right) d \tau=\alpha_{0} \int_{0}^{\infty} u_{0} F\left(u_{0}^{2}, \tau\right) d \tau .
$$

Comparing this result with the normalization condition (4.3), we see that $\alpha_{0}=1$. Thus $u_{0}(t)$ is shown to be a solution of the differential equation (1.1). In view of the uniform convergence of $\left\{u_{n}(t)\right\}$, Lemma 3.2 shows that $u_{0}(t) \rightarrow 0$ as $t \rightarrow \infty$ and $\lim _{t \rightarrow 0} t^{-1} u_{0}(t)$ exists.

5. The existence of solutions with zeros in $(0, \infty)$. Having established the existence of a fundamental solution to (1.1) on $[0, \infty)$, we can also prove that there exist similar positive solutions of (1.1) on every subinterval of the positive $t$-axis. These solutions will also approach zero at the end-points of the interval.

Indeed, for the interval $[a, \infty), a \geqq 0$, we replace $g(t, \tau)$ in (3.9) by

$$
g(t, \tau)= \begin{cases}e^{a} \bar{e}^{t} \sinh (\tau-a), & 0 \leqq a \leqq \tau<t \\ e^{a} \bar{e}^{\tau} \sinh (t-a), & t \leqq \tau\end{cases}
$$

also for the interval $[a, b], 0 \leqq a<b<\infty$, we define

$$
g(t, \tau)= \begin{cases}\frac{\sinh (b-t) \sinh (\tau-a)}{\sinh (b-a)}, & 0 \leqq a \leqq \tau<t \\ \frac{\sinh (b-\tau) \sinh (t-a)}{\sinh (b-a)}, & t \leqq \tau \leqq b .\end{cases}
$$

The corresponding variational problem for the interval $[a, b]($ or $[a, \infty)]$ will then have a solution which also solves the boundary value problem

(5.1) $\quad \ddot{y}-y+y F\left(y^{2}, t\right)=0, \begin{cases}y(a)=y(b)=0, & 0 \leqq a<b<\infty \\ y(a)=\lim _{t \rightarrow \infty} y(t)=0, & 0 \leqq a<\infty,\end{cases}$

The proof of these statements is the same as before, except that the special treatment of the singularities at $t=0, t=\infty$, or at both of these points, now becomes unnecessary. Our final result may then be stated

THEOREM 5.1. Let $\Gamma$ denote the class of functions $y(t)$ which are continuous and piecewise differentiable in $[a, b], 0 \leqq a \leqq b \leqq \infty$, satisfy $y(a)=y(b)=0, y(t) \not \equiv 0$ in $[a, b]$, and are nonnegative in $(a, b)$. Let us require, moreover, that

$$
\int_{a}^{b}\left(\dot{y}^{2}+y^{2}\right) d t=\int_{a}^{b} y^{2} F\left(y^{2}, t\right) d t
$$


where $F$ satisfies conditions (Ia)-(Ie).

Then if we define

$$
J(y)=\int_{a}^{b}\left[\dot{y}^{2}+y^{2}-G\left(y^{2}, t\right)\right] d t .
$$

where $y(t) \in \Gamma$ and $G(\eta, t)$ is given by (2.3), the minimum problem

$$
J(y)=\min .=\lambda(a, b)
$$

is solved by a solution of the differential equation (1.1). Moreover, $y(t)>0$ in $(a, b), \lambda(a, b)>0$, and if $a=0, \lim _{t \rightarrow 0} t^{-1} y(t)=0$.

We shall outline the completion of the proof of Theorem I and establish the existence of a discrete infinity of solutions $y_{1}(t), y_{2}(t), \cdots, y_{n}(t), \cdots$, in $[0, \infty)$ such that $y_{n}(0)=0, \lim _{t \rightarrow 0} t^{-1} y_{n}(t)$ exists and $\lim _{t \rightarrow \infty} y(t)=0$. Furthermore, the $n^{\text {th }}$ solution will $\stackrel{t \rightarrow 0}{b e}$ shown to possess $n \stackrel{t \rightarrow \infty}{-1}$ distinct zeros in $(0, \infty)$. We may follow a procedure due to Nehari [6] but must also take into account the nature of the singularities at zero and infinity. The procedure depends on the following result.

Letting $\lambda(a, b)$ denote the minimum of $J(y)$ in (5.3) for the interval $[a, b]$, we first prove

Lemma 5.1. (a) If $a \leqq a^{\prime} \leqq b^{\prime}<b$, then $\lambda(a, b) \leqq \lambda\left(a^{\prime}, b^{\prime}\right)$.

(b) $\lambda(a, b) \rightarrow \infty$ as $b-a \rightarrow 0$ (as $a \rightarrow \infty$ if $b=\infty$, as $b \rightarrow 0$ if $a=0)$.

(c) $\lambda(a, b)$ is a continuous function of $a$ and $b$ (of $b$ only if $a=0$, of $a$ only if $b=\infty$.).

Since $F(\eta, t)$ has the properties (Ia)-(Ic) and since condition (Id) and either (Ie) or (If) insure the existence of all integrals involved, the verification of Lemma 5.1 proceeds exactly as the proof of the corresponding lemma in [6]. It is necessary, however, to divide the proof into three stages for the intervals $[0, a][a, b],[b, \infty], 0<a<$ $b<\infty$ and use the inequalities and arguments of the previous existence theory.

Now if $0=t_{0}<t_{1}<t_{2}<\cdots<t_{n}=\infty$, where $t_{1}, \cdots, t_{n-1}$ are $\mathrm{n}-1$ distinct points in $(0, \infty)$, we consider functions $u_{\nu}(t)$ in the interval $\left[t_{\nu-1}, t_{\nu}\right]\left(\right.$ or $\left.\left[t_{n-1}, \infty\right)\right)$ which are piecewise continuously differentiable, vanish at $t_{\nu-1}$ and $t_{\nu}$, but not identically in $\left(t_{\nu-1}, t_{\nu}\right)$, and are normalized by

$$
\int_{t_{\nu-1}}^{t_{\nu}}\left(u_{\nu}^{2}+u_{\nu}^{2}\right) d t=\int_{t_{\nu-1}}^{t_{\nu}} u_{\nu}^{2} F\left(u_{\nu}^{2}, t\right) d t \quad \nu=1,2, \cdots, n .
$$

If we define $u(t)$ in $[0, \infty)$ by setting $u /(t)=u_{\nu}(t)$ in $\left[t_{\nu-1}, t_{\nu}\right], \nu=$ $1,2, \cdots, n$, then the $n^{\text {th }}$ "characteristic value" is defined by 


$$
\lambda_{n}=\min \int_{0}^{\infty}\left[\dot{u}^{2}+u^{2}-G\left(u^{2}, t\right)\right] d t
$$

where $u(t)$ ranges over the class of all functions with the properties indicated above. By Theorem 5.1, it is sufficient to consider functions $u(t)$ which coincide with solutions of (5.1) and are such that $u\left(t_{\nu-1}\right)=$ $u\left(t_{\nu}\right)=0$.

Let us define

$$
\mu=\sum_{\nu=1}^{n} \lambda\left(t_{\nu-1}, t_{\nu}\right), \quad\left(t_{0}=0, t_{n}=\infty\right) .
$$

By property (c) of Lemma 5.1, $\mu$ is continuous function of $t_{1}, t_{2}, \cdots, t_{n-1}$, and, by property (b), the values $t_{\nu}$ must be bounded away from each other and from infinity in any sequence of sets for which $\mu$ tends to its greatest lower bound. It is thus sufficient to confine the values $t_{1}, \cdots, t_{n-1}$ to a sufficiently large finite interval $[0, T]$ and, therefore, the minimum of $\mu$ is actually attained for some set of $n-1$ finite distinct values $t_{\nu}, \nu=1, \cdots, n-1$, with $0<t_{\nu-1}<t_{\nu}$.

Since the minimum of $\mu$ in (5.7) is the same as the minimum of $\int_{0}^{\infty}\left[\dot{u}^{2}+u^{2}-G\left(u^{2}, t\right)\right] d t$ under the normalizations

$$
\int_{t_{\nu-1}}^{t_{\nu}}\left(\dot{u}^{2}+u^{2}\right) d t=\int_{t_{\nu-1}}^{t_{\nu}} u^{2} F\left(u^{2}, t\right) d t, \quad \nu=1,2, \cdots, n
$$

and other specified conditions on $u(t)$, our minimum problem (5.5), (5.6) has a solution $y_{n}(t)$ which coincides in each interval $\left[t_{\nu-1}, t_{\nu}\right]$ with a solution $y(t)$ of (5.1). Morever, $y_{n}\left(t_{\nu-1}\right)=y_{n}\left(t_{\nu}\right)=0$ and $y_{n}(t)>0$ in $\left(t_{\nu-1}, t_{\nu}\right)$ because of Theorem 5.1. Accordingly, our " $n^{\text {th }}$ eigensolution" has $n-1$ distinct zeros in $(0, \infty)$ and thus we obtain a different solution $y_{n}(t)$ for different values of $n$.

Our task is now to show that this function $y_{n}(t)$ is a solution of (5.1) throughout the interval $[0, \infty)$ i.e., we wish to show that

$$
\lim _{t \rightarrow t_{\nu^{-}}} y_{n}^{\prime}(t)=\lim _{t \rightarrow t_{\nu^{+}}} y_{n}^{\prime}(t), \quad \nu=1,2, \cdots, n-1,
$$

after first requiring that $y_{n}(t)$ be positive in $\left(0, t_{1}\right)$ and change sign thereafter at each point $t_{\nu}$. This alternation of sign is possible since $-y(t)$ satisfied (5.1) whenever $y(t)$ does, and the change of sign does not affect the admissibility conditions or the value of $J(y)$.

Since $t_{1}, t_{2}, \cdots, t_{n-1}$ are all in $(0, \infty)$, we are examining the slopes of $y_{n}(t)$ at points where $F(\eta, t)$ is continuous. The proof of (5.8) follows its counter part in [6] except that $\int_{t_{\nu-1}}^{t_{\nu}}\left[\dot{y}^{2}-G\left(y^{2}, t\right)\right] d t$ is replaced by $\int_{0}^{\infty}\left[\dot{y}^{2}+y^{2}-G\left(y^{2}, t\right)\right] d t$. It is easy to see, however, that the extra term $\int_{t_{\nu-1}}^{t_{\nu}} y^{2} d t$ presents no additional difficulties. 
To summarize our results, we have the following theorem.

THEOREM 5.2. Let $\Gamma_{n}$ denote the class of functions $y(t)$ with the properties: $y(t)$ is continuous and piecewise differentiable in $[0, \infty)$; $y\left(t_{\nu}\right)=0$ and $y(t) \not \equiv 0$ in $\left(t_{\nu-1}, t_{\nu}\right)(\nu=1,2, \cdots, n)$, where the $t_{\nu}$ are numbers such that $0=t_{0}<t_{1}<t_{2}<\cdots<t_{n-1}<t_{n}=\infty$; moreover,

$$
\int_{t_{\nu-1}}^{t_{\nu}}\left(\dot{y}^{2}+y^{2}\right) d t=\int_{t_{\nu-1}}^{t_{\nu}} y^{2} F\left(y^{2}, t\right) d t, \quad \nu=1,2, \cdots, n,
$$

where $F(\eta, t)$ is subject to conditions (Ia)-(If).

If $G(\eta, t)$ is the function defined in (2.3), then the extremal problem

$$
\int_{0}^{\infty}\left[\dot{y}^{2}+y^{2}-G\left(y^{2}, t\right)\right] d t=\min =\lambda_{n}
$$

has a solution $y_{n}(t)$ whose derivative is continuous throughout $[0, \infty)$. The function $y_{n}(t)$ has exactly $n-1$ zeros in $(0, \infty)$, and is a nontrivial solution of the differential system

$$
\ddot{y}-y+y F\left(y^{2}, t\right)=0, \quad y(0)=\lim _{t \rightarrow \infty} y(t)=0,
$$

for which $\lim t^{-1} y(t)$ exists.

This result proves Theorem I.

6. The case $F\left(y^{2}, t\right)=p(t) y^{2 \alpha}$. In the existence theory of the previous sections, all of our results through Lemma 4.1 were valid when conditions (Ia)-(Id) and (If) were satisfied. In fact, we used the stronger condition (Ie) (rather than (If)) only to insure that the sequence $\left\{\dot{u}_{n}(t)\right\}$ converged uniformly in every finite interval $[0, T]$, and thus to prove that

$$
\lim _{n \rightarrow \infty} J\left(u_{n}\right)=J\left(\lim _{n \rightarrow \infty} u_{n}\right) .
$$

We can, however, circumvent this requirement of continuity of each $\dot{u}_{n}(t)$ at $t=0$ when we consider $F(\eta, t)$ in the special form

$$
F\left(y^{2}, t\right)=p(t) y^{2 \alpha} \text {. }
$$

The proof of the convergence of the comparison functions $u_{n}(t)$ to a fundamental solution is similar to one in [4] and the adaptations necessary in our case are repetitive of the arguments used in the proof of Theorem I. The following result is valid.

THEOREM 6.1. If $F\left(y^{2}, t\right)=p(t) y^{2 \alpha}$ and conditions (Ia)-(Ie) are replaced by $\left(\mathrm{Ia}^{\prime}\right)-\left(\mathrm{Ie}^{\prime}\right)$, Theorem 5.2 remains valid with the exception that $\lim _{t \rightarrow \infty} t^{-1} y(t)$ may no longer exist. Moreover the characteristic 
values assume the simpler form

$$
\lambda_{n}=\frac{\alpha}{\alpha+1} \int_{0}^{\infty}\left(\dot{y}_{n}^{2}+y_{n}^{2}\right) d t .
$$

7. Asymptotic estimates for certain eigenvalues. We shall now consider the special equation

$$
\ddot{y}-y+\frac{y^{3}}{t^{2}}=0,
$$

for which it is possible to obtain information concerning the behavior of the associated eigenvalues $\lambda_{n}$ for large values of $n$. We remark that (1.1) reduces to (7.1) for $F\left(y^{2}, t\right)=y^{2} / t^{2}$ and $F$ obviously satisfies conditions (Ia)-(Ie).

For the above equation (2.1) becomes

$$
J(y)=\int_{0}^{\infty}\left(\dot{y}^{2}+y^{2}-\frac{y^{4}}{2 t^{2}}\right) d t
$$

and, in view of the normalization

$$
\int_{t_{\nu-1}}^{t_{\nu}}\left(\dot{y}^{2}+y^{2}\right) d t=\int_{t_{\nu-1}}^{t_{\nu}} \frac{y^{4}}{t^{2}} d t, \quad \nu=1,2, \cdots, n,
$$

equation (7.2) reduces to

$$
J(y)=\frac{1}{2} \int_{0}^{\infty}\left(\dot{y}^{2}+y^{2}\right) d t .
$$

To recapitulate in terms of Theorem 5.2, equation (7.1) has a solution which is continuous in $[0, \infty)$, vanishes for $t=0, t=\infty$ and $n-1$ points in $[0, \infty)$. The function $y(t)$ is characterized by the variational problem:

If $t_{1}, t_{2}, \cdots, t_{n-1}$ are any $n-1$ values in $(0, \infty)$, satisfying $t_{\nu-1}<t_{\nu}$, $\nu=2, \cdots, n-1$, we consider functions $u(t)$ which vanish at zero, at infinity and these $n-1$ values $t_{\nu}$. Furthermore, we require $u(t)$ to be of class $D^{\prime}$, normalized by (7.3), nonnegative and not identically zero in $(0, \infty)$. The function for which

$$
\lambda_{n}=\min =\frac{1}{2} \int_{0}^{\infty}\left(\dot{y}^{2}+y^{2}\right) d t,
$$

for all choices of $t_{\nu}, \nu=1, \cdots, n-1$, is a solution of (7.1) with the properties indicated in Theorem 5.2. The value of the minimum, $\lambda_{n}$, in (7.5) we refer to as the " $n^{\text {th }}$ characteristic value" or " $n^{\text {th }}$ eigenvalue" of equation (7.1).

As $n$ increases, it is easy to see that $\lambda_{n}$ does also. Indeed, if we 
let $u(t)=y(t)$ for $0 \leqq t \leqq t_{n-1}$, and $u(t)=0$ for $t_{n-1} \leqq t<\infty$, where $y(t)$ is the solution of our problem for $\lambda_{n}$, then $u(t)$ is an admissible functien for the $(n-1)^{\text {th }}$ problem. Hence,

$$
\begin{aligned}
\lambda_{n-1} & \leqq \int_{0}^{\infty}\left[\dot{u}^{2}+u^{2}-G\left(u^{2}, t\right)\right] d t=\int_{0}^{t_{n-1}}\left[\dot{y}^{2}+y^{2}-G\left(y^{2}, t\right)\right] d t \\
& =\lambda_{n}-\int_{t_{n-1}}^{\infty}\left[\dot{y}^{2}+y^{2}-G\left(y^{2}, t\right)\right] d t \\
& =\lambda_{n}-\lambda_{1}\left(t_{n-1}, \infty\right) .
\end{aligned}
$$

Thus,

$$
\lambda_{n-1}<\lambda_{n}
$$

since $\lambda_{1}\left(t_{n-1}, \infty\right)$ is positive.

We shall now find more exact information concerning the $\lambda_{n}$ 's associated with equation (7.1).

If $u(t)$ is a competing function for the above minimum problem and $\int_{0}^{\infty}\left(\dot{u}^{2}+u^{2}\right) d t$ exists, then, as shown in $\S 2, u(t)$ may be normalized by multiplying $u(t)$ by a constant $\alpha_{\nu}$ in $\left(t_{\nu-1}, t_{\nu}\right), \nu=1,2, \cdots, n$. Then

$$
\int_{t_{\nu-1}}^{t_{\nu}}\left(\dot{u}^{2}+u^{2}\right) d t=\alpha_{\nu}^{2} \int_{t_{\nu-1}}^{t_{\nu}} \frac{u^{4}}{t^{2}} d t
$$

and $v(t)=\alpha_{\nu} u(t)$ satisfies (7.3) in $\left(t_{\nu-1}, t_{\nu}\right)$. Moreover, (7.5) shows that

$$
\lambda_{n} \leqq \frac{1}{2} \sum_{\nu=1}^{n} \int_{t_{\nu-1}}^{t_{\nu}}\left(\dot{v}^{2}+v^{2}\right) d t=\frac{1}{2} \sum_{\nu=1}^{n} \alpha_{\nu}^{2} \int_{t_{\nu-1}}^{t_{\nu}}\left(\dot{u}^{2}+u^{2}\right) d t,
$$

or, in view of (7.6),

$$
\lambda_{n} \leqq \frac{1}{2} \sum_{\nu=1}^{n} \frac{\left[\int_{t_{\nu-1}}^{t_{\nu}}\left(\dot{u}^{2}+u^{2}\right) d t\right]^{2}}{\int_{t_{\nu-1}}^{t_{\nu}} \frac{u^{4}}{t^{2}} d t} .
$$

We therefore can find an estimate from above for $\lambda_{n}$ by substituting into (7.7) any function $u(t)$ satisfying $u(0)=u(\infty)=u\left(t_{\nu}\right)=0, u(t) \not \equiv 0$ in $\left(t_{\nu-1}, t_{\nu}\right), u(t) \in D^{\prime}\left[t_{\nu-1}, t_{\nu}\right]$, for any set of numbers $t_{1}, \cdots, t_{n-1}$ in $(0, \infty)$.

Moreover, if $z(t)$ is the solution to our $n$-th minimum problem in $[0, b]$, then the function

$$
u(t)= \begin{cases}z(t), & 0 \leqq t \leqq b \\ 0, & b<t \leqq \infty\end{cases}
$$

is a competing function for the $n$-th problem in $[0, \infty)$. Hence

$$
\lambda_{n}(0, \infty) \leqq \lambda_{n}(0, b), \quad 0<b<\infty .
$$


The estimate (7.7) shall then be applied to the interval $[0, b]$, i.e., take $0=t_{0}<t_{1}<\cdots<t_{n-1}<t_{n}=b$, where $b<\infty$.

For a competing function $u(t)$, we take a solution to the differential equation

$$
\ddot{u}+u^{3}=0
$$

for which $u\left(t_{\nu-1}\right)=u\left(t_{\nu}\right)=0, u(t)>0$ in each interval $\left[t_{\nu-1}, t_{\nu}\right]$. The existence of such solutions is proved in [5].

We note that

$$
u^{3}(t)=\left[\int_{t_{\nu-1}}^{t} \dot{u}(t) d t\right]^{2} \leqq\left(t-t_{\nu-1}\right) \int_{t_{\nu-1}}^{t_{\nu}} \dot{u}^{2} d t,
$$

for $t_{\nu-1} \leqq t \leqq t_{\nu}$, and thus

$$
\int_{t_{\nu-1}}^{t_{\nu}} u^{2} d t \leqq \frac{\left(t_{\nu}-t_{\nu-1}\right)^{2}}{2} \int_{t_{\nu-1}}^{t_{\nu}} \dot{u}^{2} d t
$$

Furthermore, since

$$
\int_{t_{\nu-1}}^{t_{\nu}} \frac{u^{4}}{t^{2}} d t \geqq \frac{1}{t_{\nu}^{2}} \int_{t_{\nu-1}}^{t_{\nu}} u^{4} d t, \quad \nu=1,2, \cdots, n,
$$

the inequalities (7.7) and (7.8) show that

$$
\lambda_{n}(0, \infty) \leqq \frac{1}{2} \sum_{\nu=1}^{n} \frac{t_{\nu}^{2}\left[1+\frac{\left(t_{\nu}-t_{\nu-1}\right)^{2}}{2}\right]\left[\int_{t_{\nu-1}}^{t_{\nu}} \dot{u}^{2} d t\right]^{2}}{\int_{t_{\nu-1}}^{t_{\nu}} u^{4} d t}
$$

where $0<t_{0}<t_{1}<\cdots<t_{n}=b$ are any set of points in $(0, \infty)$. Using the fact that

$$
\int_{t_{\nu-1}}^{t_{\nu}} \dot{u}^{2} d t=\int_{t_{\nu-1}}^{t_{\nu}} u^{4} d t
$$

for every solution of (7.9) which vanishes at $t_{\nu-1}$ and $t_{\nu}$, and the property

$$
\int_{t_{\nu-1}}^{t_{\nu}} \dot{u}^{2} d t=\frac{A}{\left(t_{\nu}-t_{\nu-1}\right)^{3}}
$$

where

$$
A=\frac{2}{3}\left[\int_{0}^{1} \frac{d t}{\left(1-t^{4}\right)^{1 / 2}}\right]^{4}
$$

(this result is proved in [6]), we find that (7.11) reduces to the form

$$
\lambda_{n}(0, \infty) \leqq \frac{A}{2} \sum_{\nu=1}^{n}\left[\frac{t_{\nu}^{2}}{\left(t_{\nu}-t_{\nu-1}\right)^{3}}+\frac{t_{\nu}^{2}}{\left(t_{\nu}-t_{\nu-1}\right)}+\frac{t_{\nu}^{2}\left(t_{\nu}-t_{\nu-1}\right)}{4}\right]
$$


where $t_{n}=b, t_{0}=0$, and $t_{1}, t_{2}, \cdots, t_{n-1}$ are any $n-1$ distinct points in $(0, \infty)$.

To find a similar expression estimating $\lambda_{n}$ from below we proceed as follows.

From (7.3) and (7.10), it is found that

$$
\int_{t_{\nu-1}}^{t_{\nu}}\left(\dot{y}^{2}+y^{2}\right) d t=\int_{t_{\nu-1}}^{t_{\nu}} \frac{y^{4}}{t^{2}} d t \leqq\left(t_{\nu}-t_{\nu-1}\right) \int_{t_{\nu-1}}^{t_{\nu}} \dot{y}^{2} d t \int_{t_{\nu-1}}^{t_{\nu}} \frac{y^{2}}{t^{2}} d t
$$

where $y(t)$ is the solution of our minimum problem (7.5), and $t_{1}, t_{2}$, $\cdots, t_{n-1}$ are the corresponding zeros of $y(t)$. Thus,

$$
\int_{t_{\nu-1}}^{t_{\nu}}\left(\dot{y}^{2}+y^{2}\right) d t \leqq \frac{\left(t_{\nu}-t_{\nu-1}\right)}{t_{\nu-1}^{2}} \int_{t_{\nu-1}}^{t_{\nu}} \dot{y}^{2} d t \int_{t_{\nu-1}}^{t_{\nu}} y^{2} d t
$$

$\nu=2, \cdots, n-1$.

Furthermore, we define the function $v(t)=\alpha_{\nu} y(t)$ in each interval $\left[t_{\nu-1}, t_{\nu}\right]$, where $\alpha_{\nu}$ is to be determined by

$$
\int_{t_{\nu-1}}^{t_{\nu}} \dot{y}^{2} d t=\alpha_{\nu}^{2} \int_{t_{\nu-1}}^{t_{\nu}} y^{4} d t
$$

In this way, $v(t)$ becomes a competing function for the variational problem:

$$
J(v)=\int_{t_{\nu-1}}^{t_{\nu}}\left(\dot{v}^{2}-\frac{v^{4}}{2}\right) d t=\min =\mu\left(t_{\nu-1}, t_{\nu}\right)
$$

under the normalization

$$
\int_{t_{\nu-1}}^{t_{\nu}} \dot{v}^{2} d t=\int_{t_{\nu-1}}^{t_{\nu}} v^{4} d t
$$

The functions $v(t)$ are required to satisfy the same admissibility conditions as we required in $(2.1),(2.2)$. It is shown in [5] that the minimum $\mu\left(t_{\nu-1}, t_{\nu}\right)$ is attained when $v(t)$ is a solution $u(t)$ of (7.9), which takes the value zero at $t_{\nu-1}, t_{\nu}$ and is positive in $\left(t_{\nu-1}, t_{\nu}\right)$.

Because of (7.17) and the comments following (7.12), equation (7.16) shows that

$$
\mu\left(t_{\nu-1}, t_{\nu}\right)=\frac{1}{2} \int_{t_{\nu-1}}^{t_{\nu}} \dot{u}^{2} d t=\frac{A}{2\left(t_{\nu}-t_{\nu-1}\right)^{3}} \leqq \frac{1}{2} \int_{t_{\nu-1}}^{t_{\nu}} \dot{v}^{2} d t
$$

for every admissible function $v(t)$ satisfying (7.17). In particular using $v(t)=\alpha_{\nu} y(t)$ and noting (7.15), equation (7.18) yields the result

$$
\frac{A}{2\left(t_{\nu}-t_{\nu-1}\right)^{3}} \leqq \frac{1}{2} \int_{t_{\nu-1}}^{t_{\nu}} \dot{v}^{2} d t=\frac{\alpha_{\nu}^{2}}{2} \int_{t_{\nu-1}}^{t_{\nu}} \dot{y}^{2} d t=\frac{1}{2} \frac{\left[\int_{t_{\nu-1}}^{t_{\nu}} \dot{y}^{2} d t\right]^{2}}{\int_{t_{\nu-1}}^{t_{\nu}} y^{4} d t} .
$$


Since

$$
\int_{t_{\nu-1}}^{t_{\nu}} y^{4} d t \geqq t_{\nu-1}^{2} \int_{t_{\nu-1}}^{t_{\nu}} \frac{y^{4}}{t^{2}} d t=t_{\nu-1}^{2} \int_{t_{\nu-1}}^{t_{\nu}}\left(\dot{y}^{2}+y^{2}\right) d t,
$$

we may write this last expression in the form

$$
\frac{A t_{\nu-1}^{2}}{\left(t_{\nu}-t_{\nu-1}\right)^{3}} \leqq \frac{\left[\int_{t_{\nu-1}}^{t_{\nu}} \dot{y}^{2} d t\right]^{2}}{\int_{t_{\nu}-1}^{t_{\nu}}\left(\dot{y}+y^{2}\right) d t^{2}} .
$$

Let us now rewrite (7.14) in the following way

$$
\frac{t_{\nu-1}^{2}}{t_{\nu}-t_{\nu-1}} \leqq \frac{\int_{t_{\nu-1}}^{t_{\nu}} \dot{y}^{2} d t \int_{t_{\nu-1}}^{t_{\nu}} y^{2} d t}{\int_{t_{\nu-1}}^{t_{\nu}}\left(\dot{y}^{2}+y^{2}\right) d t} .
$$

Then if we add (7.19) and (7.20) and cancel out the common factor $\int_{t_{2-1}}^{t_{\nu}}\left(\dot{y}^{2}+y^{2}\right) d t$ in the right hand side, we obtain

$$
\frac{A t_{\nu-1}^{2}}{\left(t_{\nu}-t_{\nu-1}\right)^{3}}+\frac{t_{\nu-1}^{2}}{t_{\nu}-t_{\nu-1}} \leqq \int_{t_{\nu-1}}^{t_{\nu}} \dot{y}^{2} d t, \quad \nu=2, \cdots, n-1
$$

Finally, using the fact that

$$
\int_{t_{\nu-1}}^{t_{\nu}} \dot{y}^{2} d t \leqq \int_{t_{\nu-1}}^{t_{\nu}}\left(\dot{y}^{2}+y^{2}\right) d t
$$

equation (7.14) also yields

$$
\frac{t_{\nu-1}^{2}}{t_{\nu}-t_{\nu-1}} \leqq \int_{t_{\nu-1}}^{t_{\nu}} y^{2} d t, \quad \nu=2, \cdots, n-1 .
$$

Adding the last two equations and noting (7.5), we have, for $n \geqq 3$, the result

$$
\sum_{\nu=2}^{n-1}\left[\frac{A t_{\nu-1}^{2}}{2\left(t_{\nu}-t_{\nu-1}\right)^{3}}+\frac{t_{\nu-1}^{2}}{t_{\nu}-t_{\nu-1}}\right] \leqq \lambda_{n},
$$

where the $t_{1}, t_{2}, \cdots, t_{n-1}$ are the $n-1$ internal zeros of the " $n^{\text {th }}$ eigenfunction" $y(t)$.

In view of this inequality and the fact that we may take any values $t_{1}, t_{2}, \cdots, t_{n}$ in (7.13), we have proved the following result.

LEMmA 7.1. If $\lambda_{n}$ is the $n^{\text {th }}$ characteristic valve associated with the differential equation (7.1), for the boundary conditions $y(0)=$ $y(\infty)=0$, then, if $n \geqq 3$, 


$$
\begin{aligned}
& \liminf _{\substack{0<t_{\nu}<\infty \\
t_{\nu-1}<t_{\nu}}} \sum_{\nu=2}^{n-1}\left[\frac{A}{2} \frac{t_{\nu-1}^{2}}{\left(t_{\nu}-t_{\nu-1}\right)^{3}}+\frac{t_{\nu-1}^{2}}{t_{\nu}-t_{\nu-1}}\right] \leqq \lambda_{n} \\
& \quad \leqq \\
& \quad \liminf _{\substack{0<t_{\nu}<\infty \\
t_{\nu}<1<t_{\nu}}} \frac{A}{2} \sum_{\nu=1}^{n}\left[\frac{t_{\nu}^{2}}{\left(t_{\nu}-t_{\nu-1}\right)^{3}}+\frac{t_{\nu}^{2}}{\left(t_{\nu}-t_{\nu-1}\right)}+\frac{t_{\nu}^{2}\left(t_{\nu}-t_{\nu-1}\right)}{4}\right],
\end{aligned}
$$

where

$$
A=\frac{2}{3}\left[\int_{0}^{1} \frac{d t}{\left(1-t^{4}\right)^{1 / 2}}\right]^{4}
$$

A comparison of the upper and lower bounds in the above inequality suggests that they might be nearly equivalent asymptotically, but finding the exact minimum for either expression seems difficult.

We can, however, get an upper bound for $\lambda_{n}$ by first substituting the arithmetic means $t_{\nu}=\nu t_{n} / n$ in the right hand side. Minimizing with respect to $t_{n}$, we then obtain

$$
\lambda_{n} \leqq \frac{2 A}{9} \sqrt{\frac{2}{3}} \frac{n(2 n+1)(n+1)}{}
$$

or

$$
\lambda_{n} \leqq A_{1} n^{3}\left[1+0\left(\frac{1}{n}\right)\right], \quad A_{1}=\sqrt{\frac{2}{3}} \frac{8\left[\int_{0}^{1} \frac{d t}{\left(1-t^{4}\right)^{1 / 2}}\right]^{4}}{27}
$$

To find a lower bound for $\lambda_{n}$ in terms of $n$, we must replace our previous result (Lemma 7.1) with an expression that can be easily minimized. To do this, we see from the Rayleigh minimum principle that

$$
\left[\frac{1}{4}+\frac{\pi^{2}}{\log ^{2} \frac{t_{\nu}}{t_{\nu-1}}}\right] \int_{t_{\nu-1}}^{t_{\nu}} \frac{y^{2}}{t^{2}} d t \leqq \int_{t_{\nu-1}}^{t_{\nu}} \dot{y}^{2} d t
$$

for all functions $y(t)$ for which the integrals exist and which are piecewise differentiable in $\left[t_{\nu-1}, t_{\nu}\right]$ and vanish at the end points. Equality is achieved for the function

$$
y=\sqrt{t} \sin \left[\frac{\pi \log \frac{t}{t_{\nu-1}}}{\log \frac{t_{\nu}}{t_{\nu-1}}}\right] .
$$

If we use the above inequality when $y(t)$ is the $n^{\text {th }}$ solution of our variational problem and $t_{1}, t_{2}, \cdots, t_{n-1}$ are its internal zeros, and apply the techniques used above, we can obtain the estimate 


$$
\frac{n-2}{4}+\frac{\pi^{2}(n-2)^{3}}{\left(6 \log n+c_{1}\right)^{2}} \leqq \lambda_{n}
$$

where $c_{1}$ is a finite constant and $n \geqq 3$.

We summarize the results in (7.23) and 7.24 as follows.

THEOREM 7.1. Let $\lambda_{n}$ be the $n^{\text {th }}$ characteristic value associated with equation (7.1) and the variational problem (7.3), (7.4), (7.5). For $n \geqq 3, \lambda_{n}$ satisfies the following inequalities.

$$
\frac{\pi^{2}(n-2)^{3}}{36 \log ^{2} n}\left[1+0\left(\frac{1}{\log n}\right)\right] \leqq \lambda_{n} \leqq A_{1} n^{3}\left[1+0\left(\frac{1}{n}\right)\right],
$$

where

$$
A_{1}=\sqrt{\frac{2}{3}} \frac{8}{27}\left[\int_{0}^{1}\left[1-t^{4}\right]^{-1 / 2} d t\right]^{4}
$$

8. Some numerical results. In this section we shall obtain further information regarding the behavior of solutions to the equation

$$
\ddot{y}-y+\frac{y^{k}}{t^{k-1}}=0, \quad \text { for } k=2,3 .
$$

Synge [8] studied this equation for $k=2$ with a view to obtaining numerical values for the fundamental solution. It is our aim to verify his results and compute the fundamental solution also for $k=3$.

To do this, we note that when $F\left(y^{2}, t\right)=\left(|y|^{k-1}\right) / t^{k-1}(3.10)$ becomes

$$
u_{n}(t)=e^{-t} \int_{0}^{t} \sinh \tau \frac{y_{n}^{k}}{\tau^{k-1}} d \tau+\sinh t \int_{t}^{\infty} e^{-\tau} \frac{y_{n}^{k}}{\tau^{k-1}} d \tau
$$

$\left(\left|y_{n}\right|=y_{n}\right.$ since we consider only nonnegative functions). It was shown that $J\left(u_{n}\right) \leqq J\left(y_{n}\right)$ where equality holds only if $u_{n}(t)$ coincides with the solution of the variational problem

$$
\begin{aligned}
J(y)= & \int_{0}^{\infty}\left(\dot{y}^{2}+y^{2}-\frac{k-1}{k+1} \frac{|y|^{k+1}}{t^{k-1}}\right) d t=\min \\
& \int_{0}^{\infty}\left(\dot{y}^{2}+y^{2}\right) d t=\int_{0}^{\infty} \frac{|y|^{k+1}}{t^{k-1}} d t .
\end{aligned}
$$

We may convert our existence proof into a procedure for the numerical computation of the fundamental solution $y(t)$ by starting with a function $v_{0}(t)$, nonnegative in $(0, \infty)$, for which $v_{0}(0)=v_{0}(\infty)=0$, $v_{0}(t) \not \equiv 0, \int_{0}^{\infty}\left(\dot{v}_{0}^{2}+v_{0}^{2}\right) d t$ exists and

$$
\int_{0}^{\infty}\left(\dot{v}_{0}^{2}+v_{0}^{2}\right) d t=\int_{0}^{\infty} \frac{v_{0}^{k+1}}{t^{k-1}} d t
$$


It we then define $v_{1}(t), v_{2}(t), \cdots$, by

$$
\begin{aligned}
v_{n+1}(t)= & \alpha_{n+1} e^{-t} \int_{0}^{t} \sinh \tau \frac{v_{n}^{k}}{\tau^{k-1}} d \tau \\
& +\sinh t \int_{t}^{\infty} e^{-\tau} \frac{v_{n}^{k}}{\tau^{k-1}} d \tau
\end{aligned}
$$

where $\alpha_{n+1}$ is determined by

$$
\int_{0}^{\infty}\left(\dot{v}_{n+1}^{2}+v_{n+1}^{2}\right) d t=\int_{0}^{\infty} \frac{v_{n+1}^{k+1}}{\tau^{k-1}} d \tau
$$

we shall have $J\left(v_{n+1}\right) \leqq J\left(v_{n}\right)$. If there is a unique nonnegative solution to (8.1) for $1<k<5$, the above procedure must converge to it.

In order to test the rapidity of convergence in the above iteration, the problem was programmed for $k=2$ on a Bendix G-20 computer, using a Simpson's Rule evaluation of the integrals in (8.2). The second integral was restricted to the interval $[0,10]$ and $v(t)=$ $t e^{-1}$ was used as an initial approximation.

After 23 iterations it was found that

$$
\left|v_{n}(t)-v_{n+1}(t)\right| \leqq .00001, \quad n=23,0 \leqq t \leqq 10 .
$$

where $v_{n}(t)$ was evaluated at multiples of $\Delta t=.05$.

Setting $y(t)=v_{23}(t), \quad x(t)=t^{-1} y(t)$, the following results were obtained.

$$
\begin{aligned}
& x(0)=\dot{y}(0)=4.19172=\int_{0}^{\infty} e^{-\tau} \frac{y^{2}(\tau)}{\tau} d \tau, \\
& x(4.5)=.03926, \\
& A=16.0687=\int_{0}^{\infty} \sinh \tau \frac{y^{2}(\tau)}{\tau} d \tau,
\end{aligned}
$$

where $y(t) \sim A e^{-t}$ for large $t$ and $y(t) \sim \dot{y}(0) \sinh t$ for small values of $t$ [4]. We recall that $x(t)$ is the corresponding solution of (1.3). The values found by Synge were

$$
\begin{aligned}
& x(0)=\dot{y}(0)=4.19169 \\
& x(4.5)=.03926 \\
& A=16.0723 .
\end{aligned}
$$

A comparison of (8.4) and (8.5) shows that the correspondence is good, especially for $x(0)$ and $x(4.5)$, whereas for $A$ the correspondence occurs for one less significant digit. We thus apply the same iterative procedure outlined in (8.2) and (8.3) for the case $k=3$ and find that 


$$
\begin{aligned}
& \lambda_{1}=\frac{1}{2} \int_{0}^{\infty}\left(\dot{y}^{2}+y^{2}\right) d t=3.00787 \\
& x(0)=\dot{y}(0)=4.33738 \\
& A=2.71386=\int_{0}^{\infty} t^{-2} \sinh t y^{3}(t) d t,
\end{aligned}
$$

where, as previously noted, $y(t) \sim A e^{-t}$ for large $t$ and $y(t) \sim \dot{y}(0) \sinh t$ for small values of $t$.

It is also shown in [4] that when the values $\dot{y}(0)$ and $A$ are given, there are simpler iteration procedures, for calculating $y(t)$, which are valid at the ends of the interval.

The author wishes to express his gratitude to Professor Zeev Nehari for his invaluable advice and guidance furnished during the course of this research work.

\section{REFERENCES}

1. R. Finkelstein, R. Le Levier and M. Ruderman, Nonlinear spinor fields, Phys. Rev. 83 (1951), 326.

2. G. H. Hardy, J. E. Littlewood and G. Polya, Inequalities, Cambridge, 1952.

3. N. V. Mitskevich, The scalar field of a stationary nucleon in a non-linear theory, Soviet Physics, JETP 2 (1956), 197.

4. Z. Nehari, On a nonlinear differential equation arising in nuclear physics, Proc. Royal Irish Acad. 62 (1963).

5. - On a class of non-linear second-order differential equations, Trans. Amer. Math. Soc. 95 (1960), 101.

6. - Characteristic values associated with a class of non-linear second-order differential equations, Acta Math. 105 (1961), 141.

7. N. Rosen and H. B. Rosenstock, The forces between particles in a nonlinear field theory, Phys. Rev. 85 (1952), 257.

8. J. L. Synge, On a certain nonlinear differential equation, Proc. Royal Irish Acad. 62 (1961).

9. Y. Takahashi, The structure of the nucleon core by the Hartree approximation, Nuclear Physics 26 (1961), 658.

Received July 9, 1965 and in revised form July 9, 1966.

Montana State University

Bozeman, Montana 



\section{PACIFIC JOURNAL OF MATHEMATICS}

\section{EDITORS}

\section{H. SAMELSON}

Stanford University

Stanford, California

J. P. JANS

University of Washington

Seattle, Washington 98105
J. DUGUNDJI

University of Southern California Los Angeles, California 90007

RICHARD ARENS

University of California

Los Angeles, California 90024

\section{ASSOCIATE EDITORS}
E. F. BECKENBACH
B. H. NEumanN
F. WOLF
K. YosidA

\section{SUPPORTING INSTITUTIONS}

\author{
UNIVERSITY OF BRITISH COLUMBIA \\ CALIFORNIA INSTITUTE OF TECHNOLOGY \\ UNIVERSITY OF CALIFORNIA \\ MONTANA STATE UNIVERSITY \\ UNIVERSITY OF NEVADA \\ NEW MEXICO STATE UNIVERSITY \\ OREGON STATE UNIVERSITY \\ UNIVERSITY OF OREGON \\ OSAKA UNIVERSITY \\ UNIVERSITY OF SOUTHERN CALIFORNIA
}

\author{
STANFORD UNIVERSITY \\ UNIVERSITY OF TOKYO \\ UNIVERSITY OF UTAH \\ WASHINGTON STATE UNIVERSITY \\ UNIVERSITY OF WASHINGTON \\ AMERICAN MATHEMATICAL SOCIETY \\ CHEVRON RESEARCH CORPORATION \\ TRW SYSTEMS \\ NAVAL ORDNANCE TEST STATION
}

Mathematical papers intended for publication in the Pacific Journal of Mathematics should be typewritten (double spaced). The first paragraph or two must be capable of being used separately as a synopsis of the entire paper. It should not contain references to the bibliography. Manuscripts may be sent to any one of the four editors. All other communications to the editors should be addressed to the managing editor, Richard Arens at the University of California, Los Angeles, California 90024.

50 reprints per author of each article are furnished free of charge; additional copies may be obtained at cost in multiples of 50 .

The Pacific Journal of Mathematics is published monthly. Effective with Volume 16 the price per volume ( 3 numbers) is $\$ 8.00$; single issues, $\$ 3.00$. Special price for current issues to individual faculty members of supporting institutions and to individual members of the American Mathematical Society: $\$ 4.00$ per volume; single issues $\$ 1.50$. Back numbers are available.

Subscriptions, orders for back numbers, and changes of address should be sent to Pacific Journal of Mathematics, 103 Highland Boulevard, Berkeley 8, California.

Printed at Kokusai Bunken Insatsusha (International Academic Printing Co., Ltd.), 7-17, Fujimi 2-chome, Chiyoda-ku, Tokyo, Japan.

PUBLISHED BY PACIFIC JOURNAL OF MATHEMATICS, A NON-PROFIT CORPORATION

The Supporting Institutions listed above contribute to the cost of publication of this Journal, but they are not owners or publishers and have no responsibility for its content or policies. 


\section{Pacific Journal of Mathematics

Vol. 22, No. $3 \quad$ March, 1967

Wai-Mee Ching and James Sai-Wing Wong, Multipliers and $H^{*}$

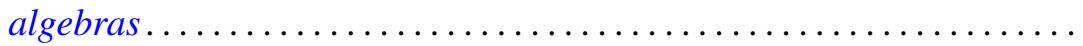

P. H. Doyle, III and John Gilbert Hocking, A generalization of the Wilder

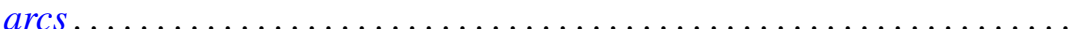

Irving Leonard Glicksberg, A Phragmén-Lindelöf theorem for function

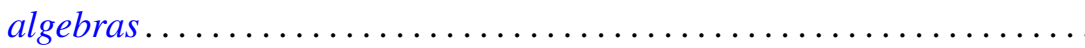

E. M. Horadam, A sum of a certain divisor function for arithmetical semi-groups ..................................... 407

V. Istrăţescu, On some hyponormal operators ................... 413

Harold H. Johnson, The non-invariance of hyperbolicity in partial

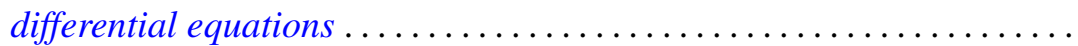

Daniel Paul Maki, On constructing distribution functions: A bounded denumerable spectrum with $n$ limit points................... 431

Ronald John Nunke, On the structure of Tor. II .................... 453

T. V. Panchapagesan, Unitary operators in Banach spaces ............. 465

Gerald H. Ryder, Boundary value problems for a class of nonlinear differential equations ................................. 477

Stephen Simons, The iterated limit condition and sequential convergence .................................... 505

Larry Eugene Snyder, Stolz angle convergence in metric spaces ......... 515 Sherman K. Stein, Factoring by subsets ................... 523

Ponnaluri Suryanarayana, The higher order differentiability of solutions of abstract evolution equations . . .

Leroy J. Warren and Henry Gilbert Bray, On the square-freeness of Fermat and Mersenne numbers ............................... 563

Tudor Zamfirescu, On l-simplicial convexity in vector spaces........... 565 Eduardo H. Zarantonello, The closure of the numerical range contains the spectrum 